\title{
Prognostic significance of the tumor-stroma ratio in gallbladder cancer
}

\author{
H. LI ${ }^{1}$, S. L. YUAN ${ }^{1}$, Z. Z. HAN ${ }^{1}$, J. HUANG ${ }^{1}$, L. CUI ${ }^{2}$, C. Q. JIANG ${ }^{2}$, Y. ZHANG ${ }^{1, *}$ \\ ${ }^{1}$ Department of Oncology, Qingdao Municipal Hospital, School of Medicine, Qingdao University, Qingdao 266011, China; ${ }^{2}$ Department of \\ Pathology, Qingdao Municipal Hospital, Qingdao 266011, China \\ *Correspondence: kadinazhang@163.com
}

Received September 15, 2016 / Accepted January 7, 2017

\begin{abstract}
In recent years, the tumor-stroma ratio (TSR) has attracted increasing attention as an independent prognostic factor for several solid tumors. However, the importance of the stromal compartment has not been investigated yet in gallbladder cancer (GBC). The objective of this study is to investigate the prognostic value of TSR in GBC and the relationship between TSR and other known prognostic parameters. A total of 51 patients who underwent operations for gallbladder carcinoma were selected for this study. TSR was determined on haematoxylin and eosin (H\&E)-stained sections by two independent investigators. Stromal ratio groups were classified as stroma-poor (ratio of stroma $<50 \%$ ) and stroma-rich (ratio of stroma $>50 \%$ ). The Mann-Whitney test, the Chi-squared test, the Kaplan-Meier method, and the Cox proportional hazards model were used to analyze the data. The median survival time for patients in the stroma-rich group was 6.00 months (95\% CI, 4.47-7.54). In contrast, for the stroma-poor group, the median survival time was 17.00 months (95\% CI, 3.64-30.36). The 3 -year overall survival rate was $19.7 \%$ in the stroma-poor group and $7.2 \%$ in the stroma-rich group. Patients with stromarich tumors had a worse prognosis than those with stroma-poor tumors (log-rank $\mathrm{P}=0.004)$. According to the univariate analysis, the TSR, differentiation grade, pTNM stage, and operative methods were shown to be related to overall survival (OS) with statistical significance. The hazard ratio (HR) of TSR was 2.428 (95\% CI, 1.29-4.58; P = 0.006). However, the TSR did not prove to be an independent prognostic factor in the multivariate analysis. Our study demonstrated that the tumor-stroma ratio (TSR) is an important prognostic parameter for gallbladder cancer (GBC). Stroma-rich tumors were associated with poor overall survival.
\end{abstract}

Key words: gallbladder cancer, tumor-stroma ratio, tumor microenvironment, prognosis

Gallbladder cancer (GBC) is an uncommon malignancy in the majority of cancer patients worldwide, but it is the most prevalent and aggressive malignancy of the biliary tract. It is a highly lethal disease and its overall 5-year survival rate does not exceed 5\% [1]. Regional nodal status and the depth of tumor invasion (T status) are the two most important prognostic factors for GBC. The histological subtype of gallbladder cancer is another important prognostic factor [2]. Currently, R0 surgical resection is the only curative treatment for GBC. Treatment of the cancer mainly depends on its clinical stage and the characteristics of the tumor cells. However, patients diagnosed at the same stage often have markedly different prognoses. Current research needs to investigate additional prognostic markers. The stromal compartment of tumors may provide valuable information about such markers. Through continuous paracrine communication, the tumor stromal compartment, consisting of fibroblasts, pericytes, leukocytes, endothelial cells, and extracellular matrix [ECM], plays an important part in tumor initiation, growth, and progression [3]. In GBC however, the importance of the stromal compartment has not been investigated.

In recent years, the components of the tumor stroma have attracted growing attention as independent prognostic factors for several solid tumors. Previous studies evaluated patients with stroma-rich tumors who were found to have a worse prognosis in cases of colon carcinoma [4], hepatocellular carcinoma [5], early cervical carcinoma [6], breast cancer [7], and epithelial ovarian cancer [8]. We hypothesize that the tumor-stroma ratio (TSR) may also be an important prognostic factor for GBC. Since the prognostic value of the TSR has not been explored for GBC, we aimed to investigate the prognostic value of TSR in GBC, as well as the relationship between TSR and other known prognostic parameters. 


\section{Patients and methods}

Patients. A total of 51 patients who underwent operations for gallbladder carcinoma at the Qingdao Municipal Hospital between January 2008 and December 2013 were selected for this study. These operations included radical operations, cholecystectomy, and palliative resection. Patients who were treated with pre-operative chemotherapy or radiotherapy were excluded, since these procedures can influence the evaluation of TSR. We used patient medical records to obtain clinical information such as gender, age, pathology type, differentiation grade, UICC T-stage, UICC N-stage, UICC M-stage, pTNM stage, surgical margin, and operative methods.

Histopathological scoring. Tissue samples obtained from the Department of Pathology included haematoxylin and eosin (H\&E)-stained sections. The most invasive region of the GBC tumor on each section was analyzed using a $4 \times$ objective. The stroma and tumor were then analyzed using a $10 \times$ objective. Only areas where both stroma and tumor were present with tumor cells found at all the borders of the image field were scored, in order to avoid scoring peripheral regions at the edge of the tumor. In cases of tumor heterogeneity, stroma-rich areas were associated with a worse prognosis and therefore considered decisive. In general, stroma-rich areas were found near areas of deepest microscopic infiltration. If the stroma tissue did not contain tumor cells, it was not thought to be related to the tumor. The assessment was performed by analyzing at least one microscopic field. The estimate was recorded as the TSR. With this protocol, two investigators independently estimated the tumor-stroma ratio in a blinded manner (per tenfold: 10, 20, $30 \%$, etc.). When the two observers differed from each other, a third pathologist was consulted. A $50 \%$ cut-off value was established as described by Mesker and colleagues [4]. Stromal ratio groups were stratified as stroma-poor: ratio of stroma < $50 \%$ and stroma-rich: ratio of stroma $>50 \%$ (Figure 1 ).

Follow-up. Follow-up data were collected until December 2015 or death. All patients were followed regularly after their operations. Overall survival (OS) was defined as the interval between the date of operation and date of death or the time of the last follow-up. Patients who were alive at the last time of follow-up were classified as censored observations.

Statistical analysis. Statistical analysis was performed using the statistical package SPSS version 17.0 (SPSS Inc., Chicago, IL, USA). The associations between TSR and other clinicopathological parameters were investigated using the Mann-Whitney test and the Chi-squared test, respectively, for continuous variables and categorical variables. The Cohen's kappa coefficient was used to assess intra-observer variability. All cases of death irrelevant to GBC were censored. The survival curves were analyzed via the Kaplan-Meier method. The log rank test was used to assess the differences between the groups for statistical significance. The Cox proportional hazards model was used to determine the hazard ratio (HR) and $95 \%$ confidence intervals (CIs) of variables for OS in univariate and multivariate analysis. Only those variables with statistical significance in univariate analysis were included in multivariate analysis. All statistical tests were 2-sided, and $\mathrm{P}<0.05$ was considered to be statistically significant.

\section{Results}

Patients and clinicopathological features. A total of 51 patients with gallbladder cancer were included in this study, with exclusion of samples involving poor histological quality, incomplete patient information, and failure of follow-up. The median age of the 51 patients was 61 years (range, 40-81) at
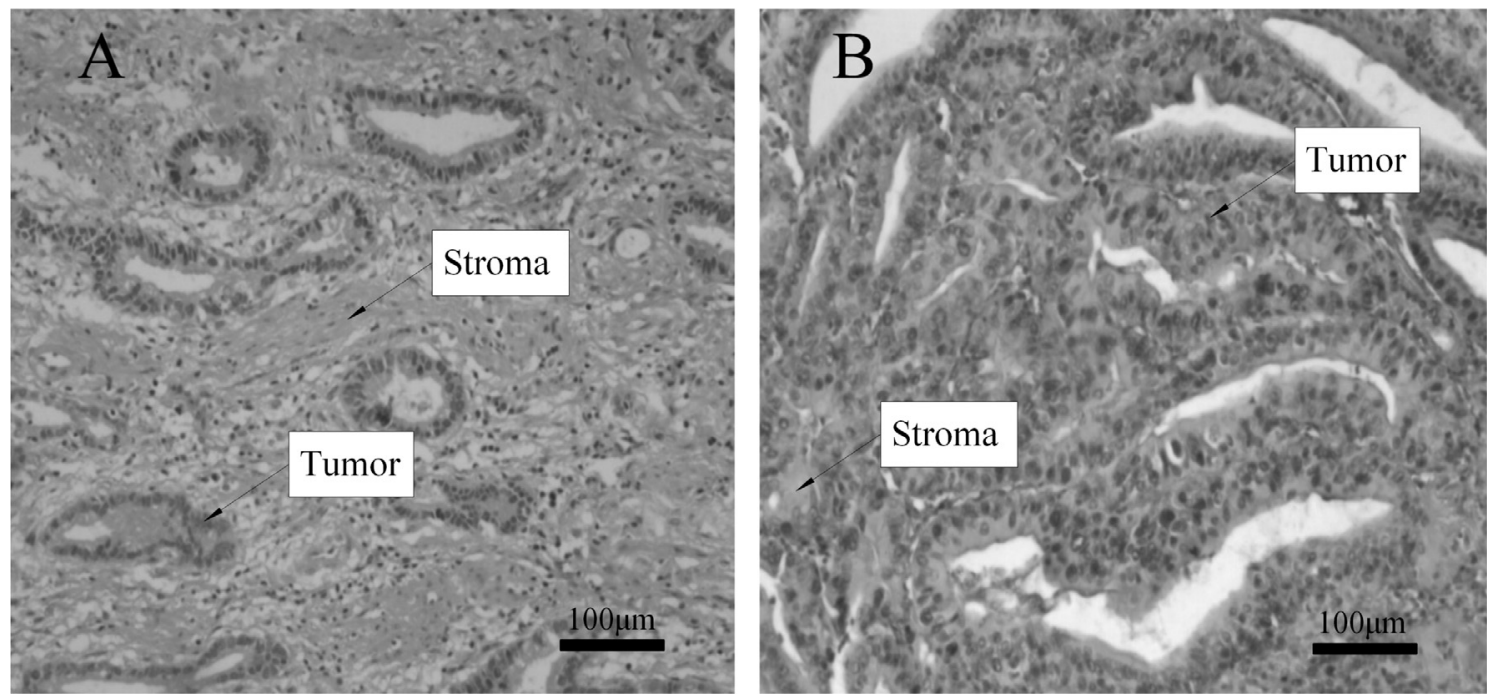

Figure 1. Haematoxylin and eosin (H\&E)-stained $5 \mu \mathrm{m}$ paraffin sections of the most invasive regions of primary gallbladder tumors. 100 $\times$ magnification (10× objective). (A) Stroma-rich $(70 \%)$ and (B) stroma-poor (10\%) regions. 


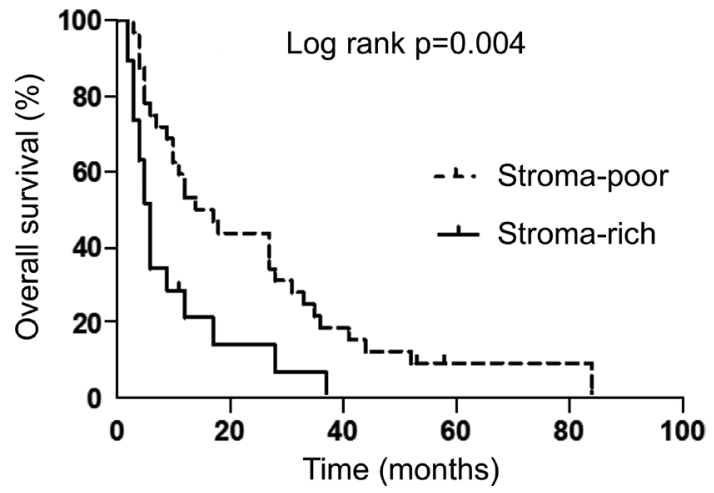

Figure 2. Kaplan-Meier curves for overall survival of 51 patients with gallbladder cancer (stroma-poor vs stroma-rich). $\log$-rank $\mathrm{P}=\mathbf{0 . 0 0 4}$.

Table 1. Clinicopathological characteristics of 51 patients with gallbladder cancer (grouped by tumor-stroma ratio).

\begin{tabular}{|c|c|c|c|c|}
\hline Characteristics & $\begin{array}{c}\text { Total } \\
(\mathrm{N}=51)\end{array}$ & $\begin{array}{l}\text { Stroma-rich } \\
\text { group } \\
(\mathrm{N}=19)\end{array}$ & $\begin{array}{c}\text { Stroma-poor } \\
\text { group } \\
(\mathrm{N}=32)\end{array}$ & $\mathrm{P}$ \\
\hline Age, years & & & & 0.876 \\
\hline Median & 61 & 65 & 61 & \\
\hline Range & $40-81$ & $43-80$ & $40-81$ & \\
\hline Sex, n & & & & 0.180 \\
\hline Male & 25 & 7 & 18 & \\
\hline Female & 26 & 12 & 14 & \\
\hline Pathology type, $n$ & & & & 1.000 \\
\hline Adenocarcinoma & 48 & 18 & 30 & \\
\hline Others & 3 & 1 & 2 & \\
\hline Differentiation grade, $\mathrm{n}$ & & & & 0.237 \\
\hline low-differentiation & 16 & 5 & 11 & \\
\hline middle-differentiation & 31 & 14 & 17 & \\
\hline high-differentiation & 4 & 0 & 4 & \\
\hline UICC T-stage, n & & & & 0.020 \\
\hline $\mathrm{T} 2$ & 5 & 0 & 5 & \\
\hline $\mathrm{T} 3$ & 32 & 10 & 22 & \\
\hline $\mathrm{T} 4$ & 14 & 9 & 5 & \\
\hline UICC N-stage, n & & & & 0.582 \\
\hline N0 & 28 & 9 & 19 & \\
\hline N1 & 13 & 5 & 8 & \\
\hline N2 & 10 & 5 & 5 & \\
\hline UICC M-stage, n & & & & 1.000 \\
\hline M0 & 48 & 18 & 30 & \\
\hline M1 & 3 & 1 & 2 & \\
\hline UICC stage, $\mathrm{n}$ & & & & 0.330 \\
\hline II & 3 & 0 & 3 & \\
\hline III & 29 & 10 & 19 & \\
\hline IV & 19 & 9 & 10 & \\
\hline surgical margin, $\mathrm{n}$ & & & & 0.535 \\
\hline negative & 46 & 16 & 30 & \\
\hline positive & 5 & 3 & 2 & \\
\hline operative methods, $\mathrm{n}$ & & & & 0.770 \\
\hline Radical operation & 26 & 7 & 19 & \\
\hline cholecystectomy & 6 & 1 & 5 & \\
\hline palliative resection & 19 & 11 & 8 & \\
\hline
\end{tabular}

the time of surgery. 48 patients were diagnosed with adenocarcinoma, 2 patients with squamous cell carcinomas, and 1 patient with adenosquamous carcinoma. Haematoxylin and eosin (H\&E)-stained sections from the most invasive part of the tumor were microscopically assessed for stromal percentages using a 10x objective. We observed areas with a size as large as one microscopic field (10x objective, 100x total magnification), as well as larger areas matching 2-4 fields, independent of the size of the tumor. The estimate of TSR was obtained and the kappa value was 0.85 , indicating good agreement between the two investigators. All inconsistent cases involved those with stromal percentages of $40-60 \% .19$ cases (37.3\%) were scored as stroma-high and 32 cases (62.7\%) were scored as stroma-low.

Association between TSR and clinicopathological features. The associations between TSR and other clinicopathological parameters were analyzed using the Mann-Whitney test for continuous variables and the Chi-squared test for categorical variables. Based on the TSR, all 51 patients were separated into a stroma-rich group $(\mathrm{n}=19)$ and a stroma-poor group $(\mathrm{n}=$ 32). Table 1 lists the clinicopathological features (gender, age, pathology type, differentiation grade, pTNM stage, surgical margin, and operative methods) grouped by tumor-stroma ratio. The results revealed that TSR was not associated with gender, age, pathology type, differentiation grade, UICC N-stage, UICC M-stage, pTNM stage, surgical margin, or operative methods, but was significantly associated with UICC T-stage $(\mathrm{P}=0.02)$ (Table 1).

Survival and multivariate analysis. A Kaplan-Meier curve was used to analyze the relationship between OS and tumorstroma ratio, which was statistically significant (log-rank $\mathrm{P}=$ 0.004 , Figure 2). Patients with stroma-rich tumors had a worse prognosis than those with stroma-poor tumors. The median survival time for patients in the stroma-rich group was 6.00 months (95\% CI, 4.47-7.54). In contrast, for the stroma-poor group, the median survival time was 17.00 months (95\% CI, $3.64-30.36$ ). The 3 -year overall survival rate was $19.7 \%$ in the stroma-poor group and $7.2 \%$ in the stroma-rich group.

The relationship between OS and all clinicopathological characteristics was investigated using the Cox univariate model. The TSR, differentiation grade, pTNM stage, and operative methods were identified as significant indicators for the OS rate. As shown in Table 2, the HR of TSR was 2.428 (95\% CI, 1.287-4.582; P =0.006). Parameters with statistical significance in the univariate analysis were included in the multivariate analysis. With the Cox multivariate analysis, only operative methods were identified as an independent prognostic factor of OS. The HR of TSR was 1.323 (95\% CI, $0.552-3.171 ; \mathrm{P}=0.530$; Table 2). Although TSR was not an independent prognostic factor for the OS rate, it was still a significant indicator for survival.

\section{Discussion}

Our study demonstrated that the tumor-stroma ratio (TSR) is an important prognostic parameter for gallbladder cancer 
(GBC). Patients with stroma-rich tumors had a worse prognosis than those with stroma-poor tumors. TSR has been shown to be a prognostic factor for GBC in previous studies. Mesker et al. were the first to demonstrate that the tumor-stroma ratio (TSR) was an independent prognostic factor for colon cancer $[4,9]$, while Huijbers et al. found that a high percentage of stromal involvement was associated with a poor prognosis. The 5-year overall survival (OS) and disease-free survival (DFS) rates were $69.0 \%$ and $58.6 \%$ versus $83.4 \%$ and $77.3 \%$ for stroma-high versus stroma-low groups, respectively [10]. Similar results can be seen in cases of hepatocellular carcinoma [5], early cervical carcinoma [6], epithelial ovarian cancer
[8], and breast cancer [7]. Our study showed that the median survival time for patients in the stroma-rich group was 6.00 months (95\% CI, 4.47-7.54). In contrast, for the stroma-poor group, the median survival time was 17.00 months $(95 \% \mathrm{CI}$, 3.64-30.36). The 3-year overall survival rate was $19.7 \%$ in the stroma-poor group and $7.2 \%$ in the stroma-rich group.

To our knowledge, the most important prognostic factors for GBC are regional nodal status, the depth of tumor invasion (T status), and the histological subtype. All these factors were analyzed in our study. TSR was significantly associated with UICC T-stage $(\mathrm{P}=0.02)$. According to the univariate analysis, the TSR, differentiation grade, pTNM stage, and operative

Table 2. Univariate Cox analysis and multivariate Cox analysis for overall survival among 51 patients with gallbladder cancer.

\begin{tabular}{|c|c|c|c|c|c|c|}
\hline \multirow{2}{*}{ Variable } & \multicolumn{3}{|c|}{ Univariate } & \multicolumn{3}{|c|}{ Multivariat } \\
\hline & HR & $95 \% \mathrm{CI}$ & $\mathrm{P}$ & HR & $95 \% \mathrm{CI}$ & $\mathrm{P}$ \\
\hline TSR & & & 0.006 & & & 0.530 \\
\hline \multicolumn{7}{|l|}{ Stroma-poor } \\
\hline Stroma-rich & 2.428 & $1.287-4.582$ & 0.006 & 1.323 & $0.552-3.171$ & 0.530 \\
\hline Age, years & & & 0.694 & & & \\
\hline Median & 0.995 & $0.969-1.021$ & 0.694 & & & \\
\hline Sex, n & & & 0.793 & & & \\
\hline \multicolumn{7}{|l|}{ Male } \\
\hline Female & 0.924 & $0.514-1.662$ & 0.793 & & & \\
\hline Pathology type, $n$ & & & 0.238 & & & \\
\hline \multicolumn{7}{|l|}{ Adenocarcinoma } \\
\hline Others & 0.488 & $0.148-1.607$ & 0.238 & & & \\
\hline differentiation grade, $\mathrm{n}$ & & & 0.002 & & & 0.075 \\
\hline \multicolumn{7}{|l|}{ low-differentiation } \\
\hline middle-differentiation & 9.002 & $2.342-34.607$ & 0.001 & 3.407 & $0.591-19.648$ & 0.170 \\
\hline high-differentiation & 3.841 & $1.112-13.268$ & 0.033 & 1.499 & $0.286-7.844$ & 0.632 \\
\hline UICC T-stage, $\mathrm{n}$ & & & $<0.001$ & & & 0.701 \\
\hline \multicolumn{7}{|l|}{$\mathrm{T} 2$} \\
\hline T3 & 0.074 & $0.019-0.289$ & $<0.001$ & 0.478 & $0.052-4.391$ & 0.514 \\
\hline $\mathrm{T} 4$ & 0.345 & $0.176-0.677$ & 0.002 & 0.990 & $0.174-5.625$ & 0.991 \\
\hline UICC N-stage, $n$ & & & 0.013 & & & 0.824 \\
\hline \multicolumn{7}{|l|}{ N0 } \\
\hline N1 & 0.328 & $0.152-0.707$ & 0.004 & 1.198 & $0.248-5.778$ & 0.822 \\
\hline $\mathrm{N} 2$ & 0.601 & $0.261-1.385$ & 0.232 & 1.335 & $0.298-5.989$ & 0.706 \\
\hline UICC M-stage, $\mathrm{n}$ & & & 0.038 & & & 0.493 \\
\hline \multicolumn{7}{|l|}{ M0 } \\
\hline M1 & 0.013 & $0.012-0.880$ & 0.038 & 0.418 & $0.034-5.072$ & 0.493 \\
\hline UICC stage, $\mathrm{n}$ & & & $<0.001$ & & & 0.484 \\
\hline \multicolumn{7}{|l|}{ II } \\
\hline III & 0.030 & $0.004-0.250$ & 0.001 & 0.133 & $0.005-3.558$ & 0.229 \\
\hline IV & 0.287 & $0.150-0.548$ & $<0.001$ & 0.484 & $0.059-4.006$ & 0.501 \\
\hline surgical margin, $\mathrm{n}$ & & & 0.059 & & & \\
\hline \multicolumn{7}{|l|}{ negative } \\
\hline positive & 0.400 & $0.155-1.035$ & 0.059 & & & \\
\hline operative methods, $\mathrm{n}$ & & & $<0.001$ & & & 0.017 \\
\hline \multicolumn{7}{|l|}{ Radical operation } \\
\hline cholecystectomy & 0.150 & $0.065-0.346$ & $<0.001$ & 0.216 & $0.074-0.663$ & 0.005 \\
\hline palliative resection & 0.085 & $0.026-0.278$ & $<0.001$ & 0.197 & $0.047-0.817$ & 0.025 \\
\hline
\end{tabular}


methods were shown to be related to OS with statistical significance. TSR might be an important prognostic parameter for gallbladder cancer. However, the TSR was not identified as an independent prognostic factor in the multivariate analysis. The most likely reason was that our study was a relatively small retrospective study of 51 patients. Moreover, this outcome could be partly explained by the difference among several histological types of GBC, which leads to different effects of the standard prognostic variables. Further researches need to be done. The determination of TSR involves a simple and convenient procedure of routine pathological examination using standard H\&E sections. The repeatability of evaluation of the TSR was also adequate. The Cohen's kappa coefficient was 0.85 , indicating good agreement between the two independent investigators. Similar conclusions were reached in previous studies of cervical carcinoma (kappa value 0.87) [6] and breast cancer (kappa value 0.80) [7]. Because of its convenience, rapid, and inexpensiveness in clinical application, TSR could be a useful tool for facilitating the collection of prognostic information of GBC. In addition, the interactions between stromal components and tumors are critical for tumor aggressiveness and thus must be seriously considered for future novel therapeutic approaches [11].

There are several theories that could explain the relationship between stroma-rich tumors and poor overall survival. It is well known that endocrine, paracrine, and cell-cell interactions between the tumor microenvironment (TME) and the tumor contribute to tumor growth. The tumor microenvironment (TME) contains cells of the immune system, fibroblasts, and adipocytes, as well as blood and lymphatic vessels, and regulates many processes such as wound healing and inflammation [12]. The tumor microenvironment (TME) can potentially influence disease prognosis and is likely an important prognostic factor for several reasons. First, the location, density, and phenotype of the different immune cells, as well as secreted chemokines and cytokines, indicate 'immune contexture' which has prognostic significance [13]. Mononuclear phagocytes are classified into two main types: classically activated macrophages (M1) and alternatively activated macrophages (M2). M1- and M2-polarized macrophages display a number of distinct features. Ma et al. showed that M1 macrophage infiltration is linked to better survival [14], whereas M2 macrophage infiltration is associated with a poor prognosis $[15,16]$. The balance between the different populations of lymphocytes guides the pro or anti-tumorigenic nature of the inflammatory response [17]. PD-L1 expressed by tumoral cells inhibits the immunosuppressive functions of $\mathrm{T}$ cells by ligation with their membrane protein PD-1. PD-L1 expression has also been linked with a worse prognosis in many patients with tumors [18]. Secondly, it has been hypothesized that a higher proportion of reactive stroma could increase the tumor burden by producing more growth factors. Altered levels of TGF- $\beta$, a main mediator of communication between the tumor and its micro-environment, can cause cancer progression by stimulation of angiogenesis, tumor immune escape, and recruitment of myofibroblasts [19]. Moreover, secreted protein acidic and rich in cysteine (SPARC), a matricellular protein that promotes the interaction between tumor cells and substrates, was reported to be strongly expressed in the stroma cells of pancreatic adenocarcinoma, non-small cell lung cancer, and colorectal carcinoma [20-22]. Changes in the stroma could thus promote the invasion and metastasis of tumors. Thirdly, cancer-associated fibroblasts (CAFs) play an important role in tumor progression by producing various growth factors, angiogenic factors, inflammatory factors, and ECM proteins [23]. Fibroblasts can promote angiogenesis through the secretion of growth factors, cytokines, and extracellular matrix proteins to facilitate the processes of tumor growth and progression [24, 25]. For example, Orimo et al. found that cancer-associated fibroblasts promote angiogenesis, tumor growth, and immune cell recruitment [26]. In addition, it has been suggested that the relative amount of desmoplastic fibrosis may help the tumor to escape from immunosurveillance [27] by its encapsulation of the malignant cells to prevent any damage [28]. Hence, the communication between the tumor and its micro-environment is a complex process, and it should be recognized that the tumor stroma plays key roles in progression and invasion of GBC. Furthermore, the interaction between cancer cells and stromal cells in the tumor microenvironment may be useful for the screening of potential candidate markers for GBC in the development of future cancer therapies [29].

In conclusion, the tumor-stroma ratio (TSR) can provide prognostic information for gallbladder cancer (GBC) patients. Stroma-rich tumors were associated with poor overall survival. Since the determination of TSR is simple, readily available, and low cost, TSR may serve as a novel prognostic histological characteristic in gallbladder cancer patients. In recent years, the tumor microenvironment has attracted increasing attention in scientific research, because of its important role in tumor invasion and metastasis. Future studies will aim to find new therapeutic targets in the tumor microenvironment.

\section{References}

[1] DUFFY A, CAPANU M, ABOU-ALFA GK, HUITZIL D, JARNAGIN W et al. Gallbladder cancer (GBC): 10-year experience at Memorial Sloan-Kettering Cancer Centre (MSKCC). J Surg Oncol 2008; 98: 485-489. https://doi.org/10.1002/ jso. 21141

[2] KANTHAN R, SENGER JL, AHMED S, KANTHAN SC. Gallbladder Cancer in the 21st Century. J Oncol 2015; 2015: 967472. https://doi.org/10.1155/2015/967472

[3] PIETRAS K, OSTMAN A. Hallmarks of cancer: interactions with the tumor stroma. Exp Cell Res 2010; 316: 1324-1331. https://doi.org/10.1016/j.yexcr.2010.02.045

[4] MESKER WE, JUNGGEBURT JM, SZUHAI K, DE HEER P, MORREAU H et al. The Carcinoma-Stromal Ratio of Colon Carcinoma Is an Independent Factor for Survival Compared to Lymph Node Status and Tumor Stage. Cell Oncol 2007; 29: 387-398. 
[5] LV Z, CAI X, WENG X, XIAO H, DU C et al. Tumor-stroma ratio is a prognostic factor for survival in hepatocellular carcinoma patients after liver resection or transplantation. Surgery 2015; 158: 142-150. https://doi.org/10.1016/j.surg.2015.02.013

[6] LIU J, LIU J, LI J, CHEN Y, GUAN X et al. Tumor-stroma ratio is an independent predictor for survival in early cervical carcinoma. Gynecol Oncol 2014; 132: 81-86. https://doi. org/10.1016/j.ygyno.2013.11.003

[7] DEKKER TJ, VAN DE VELDE CJ, VAN PELT GW, KROEP JR, JULIEN JP et al. Prognostic significance of the tumor-stroma ratio: validation study in node-negative premenopausal breast cancer patients from the EORTC perioperative chemotherapy (POP) trial (10854). Breast Cancer Res Treat 2013; 139: 371379. https://doi.org/10.1007/s10549-013-2571-5

[8] CHEN Y, ZHANG L, LIU W, LIU X. Prognostic Significance of the Tumor-Stroma Ratio in Epithelial Ovarian Cancer. Biomed Res Int 2015; 2015: 589301. https://doi.org/10.1155/2015/589301

[9] MESKER WE, LIEFERS GJ, JUNGGEBURT JM, VAN PELT GW, ALBERICI $P$ et al. Presence of a high amount of stroma and downregulation of SMAD4 predict for worse survival for stage I-II colon cancer patients. Cell Oncol 2009; 31: 169-178.

[10] HUIJBERS A, TOLLENAAR RA, V PELT GW, ZEESTRATEN EC, DUTTON S et al. The proportion of tumor-stroma as a strong prognosticator for stage II and III colon cancer patients: validation in the VICTOR trial. Ann Oncol 2013; 24 : 179-185. https://doi.org/10.1093/annonc/mds246

[11] WU J, LIANG C, CHEN M, SU W. Association between tumor-stroma ratio and prognosis in solid tumor patients: a systematic review and meta-analysis. Oncotarget 2016; 7: 68954-68965. https://doi.org/10.18632/oncotarget.12135

[12] BALKWILL FR, CAPASSO M, HAGEMANN T. The tumor microenvironment at a glance. J Cell Sci 2012; 125: 5591-5596. https://doi.org/10.1242/jcs.116392

[13] POTTIER C, WHEATHERSPOON A, RONCARATI P, LONGUESPÉE R, HERFS $M$ et al. The importance of the tumor microenvironment in the therapeutic management of cancer. Expert Rev Anticancer Ther 2015; 15: 943-954. https:// doi.org/10.1586/14737140.2015.1059279

[14] MA J, LIU L, CHE G, YU N, DAI F et al. The M1 form of tumor-associated macrophages in non-small cell lung cancer is positively associated with survival time. BMC Cancer 2010; 10: 112. https://doi.org/10.1186/1471-2407-10-112

[15] LAN C, HUANG X, LIN S, HUANG H, CAI Q et al. Expression of M2-polarized macrophages is associated with poor prognosis for advanced epithelial ovarian cancer. Technol Cancer Res Treat 2013; 12: 259-267.

[16] ZHANG B, YAO G, ZHANG Y, GAO J, YANG B et al. M2polarized tumor-associated macrophages are associated with poor prognoses resulting from accelerated lymphangiogenesis in lung adenocarcinoma. Clinics (Sao Paulo) 2011; 66: 18791886. https://doi.org/10.1590/S1807-59322011001100006
[17] VEGRAN F, APETOH L, GHIRINGHELLI F. Th9 cells: a novel CD4 T-cell subset in the immune war against cancer. Cancer Res 2015; 75: 475-479. https://doi.org/10.1158/0008-5472. CAN-14-2748

[18] RUCKI AA, ZHENG L. Pancreatic cancer stroma: understanding biology leads to new therapeutic strategies. World J Gastroenterol 2014; 20: 2237-2246. https://doi.org/10.3748/ wjg.v20.i9.2237

[19] DE WEVER O, MAREEL M. Role of tissue stroma in cancer cell invasion. J Pathol 2003; 200: 429-447. https://doi. org/10.1002/path.1398

[20] INFANTE JR, MATSUBAYASHI H, SATO N, TONASCIA J, KLEIN AP et al. Peritumoral Fibroblast SPARC Expression and Patient Outcome With Resectable Pancreatic Adenocarcinoma. J Clin Oncol 2007; 25: 319-325. https:// doi.org/10.1200/JCO.2006.07.8824

[21] KOUKOURAKIS MI, GIATROMANOLAKI A, BREKKEN RA, SIVRIDIS E, GATTER KC et al. Enhanced expression of SPARC/osteonectin in the tumor-associated stroma of nonsmall cell lung cancer is correlated with markers of hypoxia/ acidity and with poor prognosis of patients. Cancer Res 2003; 63: 5376-5380.

[22] KIM JY, JEONG D, AHN TS, KIM HJ, PARK DS et al. Expression of Secreted Protein Acidic and Rich in Cysteine in the Stroma of a Colorectal Carcinoma is Associated With Patient Prognosis. Ann Coloproctol 2013; 29: 93-99. https:// doi.org/10.3393/ac.2013.29.3.93

[23] SUGIMOTO H, MUNDEL TM, KIERAN MW, KALLURI R. Identification of fibroblast heterogeneity in the tumor microenvironment. Cancer Biol Ther 2006; 5: 1640-1646. https:// doi.org/10.4161/cbt.5.12.3354

[24] EGEBLAD M, WERB Z. New functions for the matrix metalloproteinases in cancer progression. Nat Rev Cancer 2002; 2 : 161-174. https://doi.org/10.1038/nrc745

[25] KALLURI R, ZEISBERG M. Fibroblasts in cancer. Nat Rev Cancer 2006; 6: 392-401. https://doi.org/10.1038/nrc1877

[26] ORIMO A, GUPTA PB, SGROI DC, ARENZANA-SEISDEDOS F, DELAUNAY T et al. Stromal fibroblasts present in invasive human breast carcinomas promote tumor growth and angiogenesis through elevated SDF-1/CXCL12 secretion. Cell 2005; 121: 335-348. https://doi.org/10.1016/j.cell.2005.02.034

[27] KOUNIAVSKY G, KHAIKIN M, ZVIBEL I, ZIPPEL D, BRILL $S$ et al. Stromal extracellular matrix reduces chemotherapy-induced apoptosis in colon cancer cell lines. Clin Exp Metastasis 2002; 19: 55-60. https://doi.org/10.1023/A:1013880326925

[28] LIOTTA LA, RAO CN, BARSKY SH. Tumor invasion and the extracellular matrix. Lab Invest 1983; 49: 636-649.

[29] MAO Y, KELLER ET, GARFIELD DH, SHEN K, WANG J. Stromal cells in tumor microenvironment and breast cancer. Cancer Metastasis Rev 2013; 32: 303-315. https://doi. org/10.1007/s10555-012-9415-3 